Animal Health Research Institute, Damanhur, Branch.

\title{
BIOCHEMICAL CHANGES ASSOCIATED WITH ADMINISTRATION OF NON-STEROIDAL ANTI- INFLAMMATORY PIROXICAM IN BREEDING RABBIT
}

(With 5 Tables)

By

HANA EL-HOFY RASHAD and NAHID ZAGLOL*

* Animal Health Research Institute, Alex. Branch.

(Received at 14/9/2011)

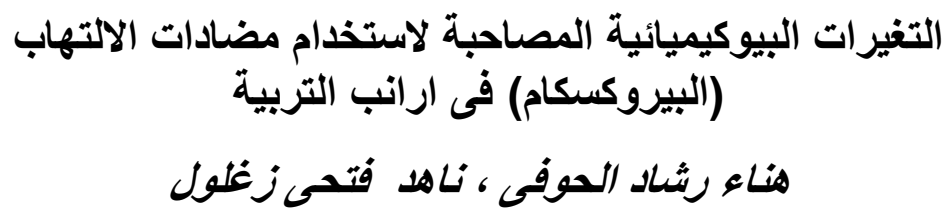

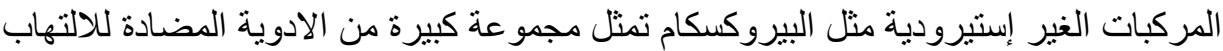

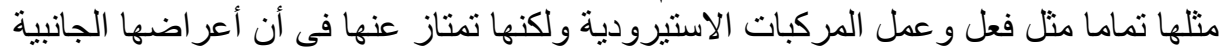

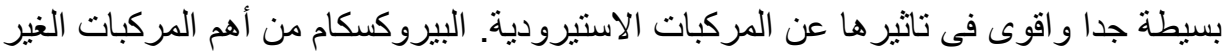

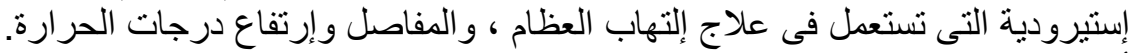

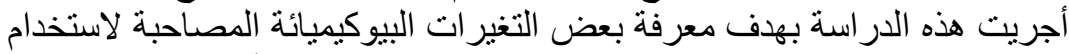

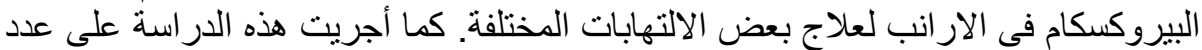

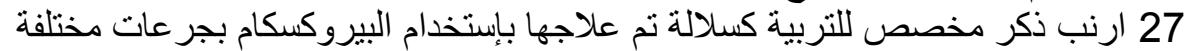

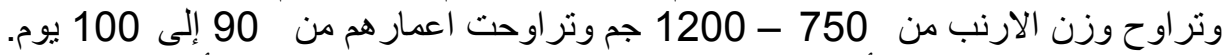

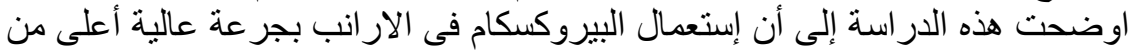

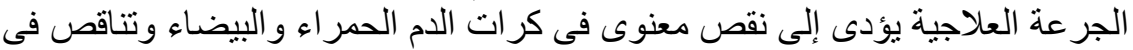

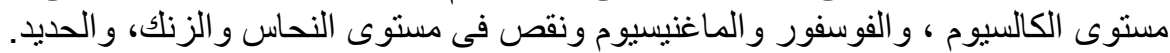

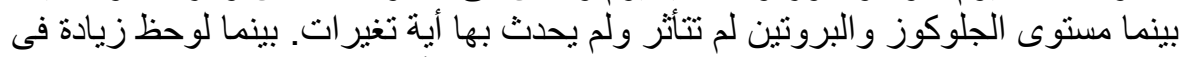

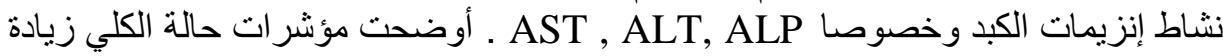

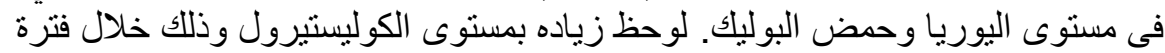

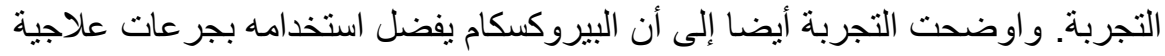
صغيرة وليس بجر عات عالية لتجنب الاعر اض البئل الجانبية له.

\section{SUMMARY}

Non-steroidal Anti-inflammatory Drugs (NSAI Ds); are chemically heterogeneous large groups of drugs which suppress inflammation in a manner similar to steroids, but with less side effects of sedation, respiratory depression, or addiction than steroids. Piroxicam is used in 
the treatment of osteoarthritis, dysmenorrhoea, and pyrexia and as an analgesic. Piroxicam selectively inhibit cyclooxygenase-2 over than cyclooxygenase- 1 enzymes. This work aimed to study the biochemical changes associated with administration of non-steroidal antiinflammatory Piroxicam in breeding rabbits. The present work was carried-out on 27 apparently healthy native male rabbits about 3 months' age and 975 gm average weight. Results showed significant decrease in $\mathrm{RBCs}, \mathrm{Hb}$ and PCV values. On the same, sever and significant decrease in total WBCs and neutrophils when injected the anti-inflammatory by over dose of the drug at all the three periods of experiment. There was significant decrease in Ca. Ph., Mag. levels .Over dose resulted in severe decrease in above elements. Also significant decrease of the copper, zinc and iron especially at the over dose Compared with control group. Blood glucose and serum protein showed non-significant changes, compared to control group. On the other hand there was a significant increase in the activities of Aspartate amino transferase (AST), Alanine aminotransferase (ALT) and Alkaline phosphatase (ALP) especially over dose of the drug. Kidney function tests showed a significant increase in both urea and creatinine especially in over dose treatment group. Also, the cholesterol level appeared as in high level in all treated group all over the period of research. Significant increase of alkaline phosphatase activities and zinc in both therapeutic and over dose along the course of the study were observed except the gradual decrease of the zinc in the testes homogenate. Nephro, hepato- and hemato-toxisity could induced by piroxicam treatment of rabbits specially by over dose ( $1 \mathrm{mg} / \mathrm{kg} . \mathrm{bw}$.) which should be avoided in field treatments of animals (rabbits)

Key words: Piroxican, drugs, non steroids, anti-inflammatory, rabbits.

\section{INTRODUCTION}

Piroxicam is 4-Hydroxy-2-methyl-N-2 pyridinyl-2H-1, 2benzothiazine-3-carboxamide 1, 1-dioxide. Oxicam members of the oxicam family are not carboxylic, acids, but they are acidic by virtue of the enolic4-hydroxy substitute (Heeb et al., 2005).

Piroxicam occurs as a white crystalline solid, sparingly soluble in water, dilute acid and most organic solvents (Sigurdardottir et al., 2008). It is highly soluble in alcohol and in aqueous solutions. It exhibits a weakly acidic-4-hydroxy proton (PKa 5.1) and weakly basic pyridyl nitrogen (PKa 1.8). The molecular weight of Piroxicam is 331.35 (Ritland and Gendler, 1999). 
Piroxicam is a non-steroidal anti-inflammatory drug (NSAIDs) that exhibits anti-inflammatory, analgesic, and antipyretic activities in animal models. The mechanism of action of Piroxicam, like that of other NSAIDs, is not completely understood but may be related to prostaglandin synthetase inhibition (Bradshaw et al., 1984).

Prostaglandins are a group of biologically active compounds with a plethora of different actions and produced in virtually all tissues of the body. They are produced on response to a great variety of stimuli. They have a major role in the mediation and modulation of inflammatory states. Many drugs which can be bought over the counter in pharmacies are anti-inflammatory agents, from lowly aspirin to many more modern remedies. All these so-called NSAIDs have their effects by reducing or preventing the actions of prostaglandins (Kocaoğlu et al., 1997; Lobetti and Joubert, 2000).

Anti-inflammatory undergo a complex series of biochemical transformation to inhibited the prostaglandins (Grosman, 2007; Martini et al., 2008).

\section{MATERIALS and METHODS}

\section{Materials:}

\subsection{Animals:}

The present work was carried-out on 27 breeding male rabbits of native strain. Their body weights ranged from 750 to $1200 \mathrm{~g}$ and their ages ranged from 90-100 days. All animals were subjected to acclimatization for two weeks.

\subsection{Chemical:}

Piroxicam is 4-Hydroxy-2-methyl-N-2 Pyridinyl-2H-1, 2benzothiazine-3-carboxamide 1, -dioxide, an oxicam.

\section{Piroxicam:}

Molecular formula $\quad \mathrm{C}_{\mathbf{1 5}} \mathrm{H}_{13} \mathrm{~N}_{\mathbf{3}} \mathrm{O}_{4} \mathrm{~S}$

The structure formula

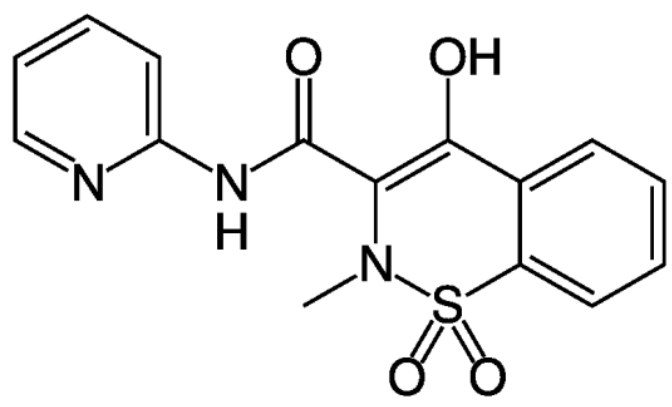




\subsection{Experimental design:}

This study was carried out for 8 successive weeks. The rabbits were classified into

(3) Groups of (9 rabbits) each as follows:

Group-1 (Control group): The rabbits were intramuscular) injected with $1 \mathrm{ml}$ normal saline once a day for 3 successive days at the

Group II and Group III where the rabbits were intramuscular injected with $0.4 \mathrm{mg}$ of active principal of piroxicam/ $\mathrm{kg}$. bw. (therapeutic dose) and $1 \mathrm{mg} / \mathrm{kg}$ bw (over dose) the drug was injected once a day for three successive days, each dose were dissolved in $1 \mathrm{ml}$ saline at the $2^{\text {nd }}, 4^{\text {th }}$ and $8^{\text {th }}$ week of the experiment, 3 rabbits were sacrificing at each period for collection of the samples.

\section{Methods:-}

\section{-Blood samples:-}

Twenty seven blood samples were collected by sacrificing of both treated and control rabbits. Nine blood samples were collected each period of experiment ( 2 nd week, $4^{\text {th }}$ week and $8^{\text {th }}$ week). Each blood samples was divide into two portions. The first was collected on EDTA) as anticoagulant for determination of hematological picture (Coles, 1986). The second portion of blood sample was collected without anticoagulant for sera separation for biochemical assays.

\section{- Clinico-biochemical analysis:}

The concentration of :serum calcium, phosphorus and magnesium ( AOAC, 1975) and serum glucose (Trinder, 1969) Total protein (Peters, 1968) Urea (Coulombe and Favreau, 1963) Creatinin (Williams, 1999) and the activity of serum ALT \& AST (Reitman and Frankel 1957) Alkaline phosphatase (Alp) (Kind and King, 1954) Enzymes were spectrophotometrically determined. Also the concentration of serum zinc, cupper and iron were determined using atomic absorption spectrophotometry (Schrenk, 1975)

\section{- Testes homogenates:}

Two weeks, $4^{\text {th }}$ weeks and $8^{\text {th }}$ weeks after the injection of antiinflammatory drugs of both therapeutic and over dose as well as control, rabbits were slaughtered and the testes were removed and kept frozen at $-20^{\circ} \mathrm{C}$ until homogenized. Ten percent testicular homogenate was prepared by homogenizing $0.5 \mathrm{~g}$ testicular tissue in $5 \mathrm{ml} 0.154 \mathrm{MKcL}$ in electrical homogenizer. The homogenate was centrifuged at $3000 \mathrm{rpm}$ for 30 minutes and the supernatant was kept frozen at $-20^{\circ} \mathrm{C}$ until assayed for enzymes activity (Alkline phosphatase) and zinc content. 
Tissue-Alkaline phosphatase in testes homogenates was estimated according to modified method of Kind and King (1954). -The concentrations of heavy metal as zinc in homogenates were determined by using Flame Atomic Absorption spectrophotometer (Perkin Elmer mode, Spectra-AA10, USA). Accurately, the apparatus was adjusted at wave length of $213.9 \mathrm{~nm}$ for zinc.

\section{Hormonal assessment:}

\section{- Testosterone:-}

Testosterone concentration were determined by radioimmunoassay described by Adams et al. (1994) using testosterone solid kits supplied by Bioscource Europe S. A. Product Line Medgenix Diagnostic Lot-no, 000501.

\section{a- Free testosterone:}

The measurement of testosterone was carried-out according to the method described by (Adams et al., 1994). The purpose of free testosterone (FT) measurement is to correct the total testosterone concentration for the effect of variable binding by Sex Hormone Binding Globulin (SHBG). It would appear, therefore, to be more appropriate to measure FT rather than TT when investigating for hypoandrogenicity. Methods available to measure FT can be complex (equilibrium dialysis and calculated free testosterone (CFT) or simple (The commercial FT kit "Coat-A-Count" using an analog tracer).

\section{b- Prostaglandin F2 alpha:}

The measurement of $\mathrm{PGF} 2_{\alpha}$ the measurement of $\mathrm{PGF}_{2 \alpha}$ was carried-out according to the method described by (Vane and Botting, 1997). Radio immunological measurement of prostaglandin (PG) $E_{2}$ and $\mathrm{F}_{2}$ alpha where $50 \mu \mathrm{L}$ serum samples are extracted with an organic solvent system. The overall recovery after extraction and purification, calculated with labeled as well as unlabeled compounds, is in the order of $70 \%$. The column elutes are assayed at 1: $12-1: 60$ dilution in the standard diluents" of the assay: $8 \mathrm{pg} \mathrm{PGF}_{2}$ alpha / $\mathrm{ml}$ of whole serum represents the lowest measurable concentration. A serum blank and a solvent blank were evaluated separately, subjecting $50 \mu \mathrm{L}$ of serum obtained from an Piroxicam ${ }^{\circledR}$ treated animal ("PG-free serum) or $50 \mu \mathrm{L}$ of distilled water, respectively, to the extraction-purification procedures (Vane and Botting, 1997). Both were found not to interfere with antigen-antibody reaction. Serum PG-like immune-reactivity (LI) was characterized in terms of immunochemical and thin-layer chromatography (TLC) behavior (Vane and Botting, 1997). 


\section{- Statistical analysis:}

The statistical analysis was carried out by the method depending on (ANOVA) multiple F. test for analysis of randomized complete design (RCBD) as multiple range test (Duncan, 1955) to study the effect of the different treatment groups on the different variables (hematological, minerals, protein and serum enzymes) under the study at different period of experiments, according to the computerized Statistical Analysis System SAS (2006).

\section{RESULTS}

With respect of hematological values (Table, 1) showed significant decrease in RBCs, Hb. and PCV values. The severe drop of these values were cleared by over therapeutic dose of piroxicam. On the same, sever and significant decrease in total WBCs and neutrophils when injected the anti-inflammatory by over dose of the periods of experiment (Table 1).

Regarding to serum calcium, phosphorus and magnesium values (Table 2) showed significant decrease in these elements. The data illustrate the injection of Piroxicam by over dose resulted to severe decrease in these elements followed by therapeutic dose. On the other hand after experimental injection of anti-inflammatory drugs leads to significant decrease of the copper, zinc and iron especially at over dose followed by therapeutic one compared with control group (Table 2). With regard to liver and kidney function tests (Table 3). Blood glucose and serum protein showed non-significant changes, compared to control group. On the other hand there were a significant increase in the activities of AST, ALT and ALP specially by over dose of the drug.

Kidney function tests showed a significant increase in both urea and creatinine especially by the over dose treatment group (Table 3). Also, the cholesterol level significantly increased in all treated group all over the three periods. Significant increase of alkaline phosphatase activities and zinc in both therapeutic and over dose along the course of the study were observed except the gradual decrease of the zinc in the testes homogenate (Table 4). 
Table 1: Showing the effects of administration of therapeutic dose $(0.4 \mathrm{mg} / \mathrm{kg} . \mathrm{bw}$.$) and over dose$ $(1 \mathrm{mg} / \mathrm{kg} . \mathrm{bw})$ of non steroidal anti-inflammatory Piroxicam ${ }^{\circledR}$ in serum of breeding rabbit on hematological picture (Mean \pm S.E.).

\begin{tabular}{|c|c|c|c|c|c|c|c|c|c|}
\hline \multirow{3}{*}{ Parameters } & \multicolumn{9}{|c|}{ Dose and periods } \\
\hline & \multicolumn{3}{|c|}{ Therapeutic dose } & \multicolumn{3}{|c|}{ Over dose } & \multicolumn{3}{|c|}{ Control } \\
\hline & $2^{\text {nd }}$ week & $4^{\text {th }}$ week & $8^{\text {th }}$ week & $2^{\text {nd }}$ week & $4^{\text {th }}$ week & $8^{\text {th }}$ week & $2^{\text {nd }}$ week & $4^{\text {th }}$ week & $8^{\text {th }}$ week \\
\hline $\begin{array}{l}\text { Total RBCs } \\
\left(\mathrm{X} 10^{6} / \mu \mathrm{L}\right)\end{array}$ & $\begin{array}{c}\text { B } \\
4.67 \pm 0.1 \\
\end{array}$ & $\begin{array}{c}\text { B } \\
4.53 \pm 0.2 \\
\end{array}$ & $\begin{array}{c}\text { B } \\
4.62 \pm 0.1 \\
\end{array}$ & $\begin{array}{c}\mathrm{C} \\
4.38 \pm 0.1 \\
\end{array}$ & $\begin{array}{c}\mathrm{C} \\
4.42 \pm 0.1 \\
\end{array}$ & $\begin{array}{c}\mathrm{C} \\
4.36 \pm 0.1 \\
\end{array}$ & $\begin{array}{c}\mathrm{A} \\
5.21 \pm 0.21 \\
\end{array}$ & $\begin{array}{c}\mathrm{A} \\
5.04 \pm 0.21 \\
\end{array}$ & $\begin{array}{c}\mathrm{A} \\
5.26 \pm 0.19\end{array}$ \\
\hline PCV \% & $\begin{array}{c}\text { B } \\
22.45 \pm 0.7 \\
\end{array}$ & $\begin{array}{c}\text { B } \\
22.06 \pm 0.61 \\
\end{array}$ & $\begin{array}{c}\text { B } \\
22.43 \pm 0.51 \\
\end{array}$ & $\begin{array}{c}\text { B } \\
22.11 \pm 0.3 \\
\end{array}$ & $\begin{array}{c}\text { B } \\
22.15 \pm 0.5\end{array}$ & $\begin{array}{c}\mathrm{C} \\
22.09 \pm 0.4 \\
\end{array}$ & $\begin{array}{c}\mathrm{A} \\
29.0 \pm 0.45 \\
\end{array}$ & $\begin{array}{c}\mathrm{A} \\
29.2 \pm 0.33\end{array}$ & $\begin{array}{c}\mathrm{A} \\
29.5 \pm 0.33\end{array}$ \\
\hline $\mathrm{Hb}(\mathrm{gm} / \mathrm{dL})$ & $\begin{array}{c}\mathrm{B} \\
6.42 \pm 0.22\end{array}$ & $\begin{array}{c}\mathrm{B} \\
6.55 \pm 0.33 \\
\end{array}$ & $\begin{array}{c}\mathrm{B} \\
6.63 \pm 0.31 \\
\end{array}$ & $\begin{array}{c}\text { B } \\
6.24 \pm 0.31\end{array}$ & $\begin{array}{c}\mathrm{C} \\
6.28 \pm 0.31\end{array}$ & $\begin{array}{c}\mathrm{C} \\
6.18 \pm 0.31\end{array}$ & $\begin{array}{c}\mathrm{A} \\
9.35 \pm 0.13\end{array}$ & $\begin{array}{c}\mathrm{A} \\
9.21 \pm 0.09\end{array}$ & $\begin{array}{c}\mathrm{A} \\
9.52 \pm 0.21\end{array}$ \\
\hline $\begin{array}{l}\text { Total WBCs } \\
\left(\mathrm{X} 10^{3} / \mu \mathrm{L}\right)\end{array}$ & $\begin{array}{c}\text { B } \\
8.41 \pm 0.81 \\
\end{array}$ & $\begin{array}{c}\mathrm{B} \\
8.98 \pm 1.03 \\
\end{array}$ & $\begin{array}{c}\text { B } \\
8.39 \pm 0.83 \\
\end{array}$ & $\begin{array}{c}\mathrm{C} \\
7.95 \pm 1.21 \\
\end{array}$ & $\begin{array}{c}\mathrm{C} \\
7.57 \pm 1.2 \\
\end{array}$ & $\begin{array}{c}\mathrm{C} \\
6.58 \pm 0.93 \\
\end{array}$ & $\begin{array}{c}\mathrm{A} \\
9.95 \pm 1.16 \\
\end{array}$ & $\begin{array}{c}\mathrm{A} \\
9.33 \pm 1.33 \\
\end{array}$ & $\begin{array}{c}\mathrm{A} \\
9.84 \pm 1.42 \\
\end{array}$ \\
\hline Neutrophil \% & $\begin{array}{c}\mathrm{A} \\
46.5 \pm 1.12\end{array}$ & $\begin{array}{c}\mathrm{A} \\
43.9 \pm 0.85 \\
\end{array}$ & $\begin{array}{c}\mathrm{A} \\
41.89 \pm 0.26 \\
\end{array}$ & $\begin{array}{c}\mathrm{C} \\
29.4 \pm 1.41 \\
\end{array}$ & $\begin{array}{c}\mathrm{C} \\
31.3 \pm 1.32 \\
\end{array}$ & $\begin{array}{c}\mathrm{B} \\
34.2 \pm 1.21 \\
\end{array}$ & $\begin{array}{c}\mathrm{A} \\
41.55 \pm 1.12 \\
\end{array}$ & $\begin{array}{c}\mathrm{A} \\
42.8 \pm 0.92 \\
\end{array}$ & $\begin{array}{c}\mathrm{A} \\
41.9 \pm 1.21 \\
\end{array}$ \\
\hline Lymphocyte \% & $\begin{array}{c}\text { B } \\
42.9 \pm 0.93\end{array}$ & $\begin{array}{c}\text { B } \\
44.5 \pm 1.31 \\
\end{array}$ & $\begin{array}{c}\mathrm{B} \\
45.2 \pm 1.21\end{array}$ & $\begin{array}{c}\mathrm{A} \\
58.7 \pm 1.41\end{array}$ & $\begin{array}{c}\mathrm{A} \\
54.2 \pm 1.38\end{array}$ & $\begin{array}{c}\mathrm{A} \\
53.1 \pm 1.72\end{array}$ & $\begin{array}{c}\mathrm{C} \\
40.9 \pm 0.87\end{array}$ & $\begin{array}{c}\mathrm{B} \\
42.7 \pm 1.21\end{array}$ & $\begin{array}{c}\mathrm{B} \\
41.2 \pm 1.23\end{array}$ \\
\hline Basophil \% & $\begin{array}{c}\mathrm{C} \\
0.7 \pm 0.11 \\
\end{array}$ & $\begin{array}{c}\mathrm{C} \\
1.36 \pm 0.32 \\
\end{array}$ & $\begin{array}{c}\text { B } \\
2.18 \pm 0.52 \\
\end{array}$ & $\begin{array}{c}\mathrm{C} \\
0.6 \pm 0.12 \\
\end{array}$ & $\begin{array}{c}\text { B } \\
2.6 \pm 0.61\end{array}$ & $\begin{array}{c}\mathrm{A} \\
3.2 \pm 0.83\end{array}$ & $\begin{array}{c}\mathrm{A} \\
2.78 \pm 0.55\end{array}$ & $\begin{array}{c}\mathrm{B} \\
3.8 \pm 0.85\end{array}$ & $\begin{array}{c}\mathrm{A} \\
2.9 \pm 0.75\end{array}$ \\
\hline Monocyte \% & $\begin{array}{c}\mathrm{B} \\
5.3 \pm 0.52\end{array}$ & $\begin{array}{c}\text { B } \\
6.21 \pm 0.84\end{array}$ & $\begin{array}{c}\mathrm{B} \\
5.2 \pm 0.61\end{array}$ & $\begin{array}{c}\mathrm{B} \\
5.1 \pm 0.79\end{array}$ & $\begin{array}{c}\mathrm{C} \\
4.7 \pm 0.35\end{array}$ & $\begin{array}{c}\mathrm{C} \\
4.3 \pm 0.27\end{array}$ & $\begin{array}{c}\mathrm{A} \\
6.5 \pm 0.82\end{array}$ & $\begin{array}{c}\mathrm{A} \\
6.2 \pm 0.75\end{array}$ & $\begin{array}{c}\mathrm{A} \\
8.1 \pm 0.92\end{array}$ \\
\hline Eosinophil \% & $\begin{array}{c}\mathrm{C} \\
4.6 \pm 0.41\end{array}$ & $\begin{array}{c}\mathrm{C} \\
4.03 \pm 0.55\end{array}$ & $\begin{array}{c}\mathrm{B} \\
5.53 \pm 0.61\end{array}$ & $\begin{array}{c}A \\
6.20 \pm 0.61\end{array}$ & $\begin{array}{c}\mathrm{A} \\
7.2 \pm 0.82\end{array}$ & $\begin{array}{c}\mathrm{B} \\
5.2 \pm 0.61\end{array}$ & $\begin{array}{c}\mathrm{A} \\
8.27 \pm 1.11\end{array}$ & $\begin{array}{c}\text { B } \\
4.5 \pm 0.93\end{array}$ & $\begin{array}{c}\mathrm{B} \\
5.9 \pm 0.59\end{array}$ \\
\hline
\end{tabular}

For each week: Means within the same row of different litters are significantly different. 
Table 2: Showing the effects of administration of therapeutic dose $((0.4 \mathrm{mg} / \mathrm{kg} . \mathrm{bw}$.$) and over dose$ $(1 \mathrm{mg} / \mathrm{kg} . \mathrm{bw}))$ of non steroidal anti-inflammatory Piroxicam ${ }^{\circledR}$ in serum of breeding rabbit on macro and microminerals (Mean \pm S.E.).

\begin{tabular}{|c|c|c|c|c|c|c|c|c|c|}
\hline \multirow{3}{*}{ Parameters } & \multicolumn{9}{|c|}{ Dose and periods } \\
\hline & \multicolumn{3}{|c|}{ Therapeutic dose } & \multicolumn{3}{|c|}{ Over dose } & \multicolumn{3}{|c|}{ Control } \\
\hline & $2^{\text {nd }}$ week & $4^{\text {th }}$ week & $8^{\text {th }} \mathrm{w}$ & $2^{\text {nd }}$ week & $4^{\text {th }}$ week & $8^{\text {th }}$ & $2^{\text {nd }}$ week & $4^{\text {th }}$ week & $8^{\text {th }} v$ \\
\hline $\begin{array}{c}\text { Calcium } \\
\text { (mg/dl) }\end{array}$ & $\begin{array}{c}\text { B } \\
5.24 \pm 0.23 \\
\end{array}$ & $\begin{array}{c}\text { B } \\
5.4 \pm 0.11 \\
\end{array}$ & $\begin{array}{c}\text { B } \\
5.21 \pm 0.33 \\
\end{array}$ & $\begin{array}{c}\mathrm{C} \\
4.51 \pm 0.33 \\
\end{array}$ & $\begin{array}{c}\mathrm{C} \\
4.13 \pm 0.21 \\
\end{array}$ & $\begin{array}{c}\mathrm{C} \\
3.58 \pm 0.2 \\
\end{array}$ & $\begin{array}{c}\mathrm{A} \\
9.45 \pm 0.19 \\
\end{array}$ & $\begin{array}{c}\mathrm{A} \\
10.23 \pm 0.21\end{array}$ & $\begin{array}{c}\mathrm{A} \\
10.16 \pm 0.33\end{array}$ \\
\hline $\begin{array}{c}\text { Phosphorus } \\
(\mathrm{mg} / \mathrm{dl})\end{array}$ & $\begin{array}{c}\text { B } \\
4.12 \pm 0.11 \\
\end{array}$ & $\begin{array}{c}\text { B } \\
4.63 \pm 0.20 \\
\end{array}$ & $\begin{array}{c}\text { B } \\
4.45 \pm 0.23 \\
\end{array}$ & $\begin{array}{c}\mathrm{C} \\
2.87 \pm 0.06 \\
\end{array}$ & $\begin{array}{c}\mathrm{C} \\
2.89 \pm 0.09 \\
\end{array}$ & $\begin{array}{c}\mathrm{C} \\
2.55 \pm 0.06 \\
\end{array}$ & $\begin{array}{c}\text { A } \\
6.12 \pm 0.54 \\
\end{array}$ & $\begin{array}{c}\text { A } \\
6.23 \pm 0.31 \\
\end{array}$ & $\begin{array}{c}\mathrm{A} \\
6.44 \pm 0.09 \\
\end{array}$ \\
\hline $\begin{array}{l}\text { Magnesiu } \\
\mathrm{m}(\mathrm{mg} / \mathrm{dl})\end{array}$ & $\begin{array}{c}\text { B } \\
2.21 \pm 0.08 \\
\end{array}$ & $\begin{array}{c}\text { B } \\
2.34 \pm 0.09 \\
\end{array}$ & $\begin{array}{c}\text { B } \\
2.31 \pm 0.06 \\
\end{array}$ & $\begin{array}{c}\mathrm{C} \\
1.37 \pm 0.07 \\
\end{array}$ & $\begin{array}{c}\mathrm{C} \\
1.0 \pm 0.09 \\
\end{array}$ & $\begin{array}{c}\mathrm{C} \\
2.48 \pm 0.07 \\
\end{array}$ & $\begin{array}{c}\mathrm{A} \\
2.95 \pm 0.05 \\
\end{array}$ & $\begin{array}{c}\mathrm{A} \\
3.85 \pm 0.03 \\
\end{array}$ & $\begin{array}{c}\mathrm{A} \\
3.93 \pm 0.09 \\
\end{array}$ \\
\hline $\begin{array}{l}\text { Copper } \\
(\mu \mathrm{g} / \mathrm{dl})\end{array}$ & $\begin{array}{c}\text { B } \\
130.14 \pm 1.21 \\
\end{array}$ & $\begin{array}{c}\text { B } \\
125.23 \pm 2.35 \\
\end{array}$ & $\begin{array}{c}\text { B } \\
128.50 \pm 2.13 \\
\end{array}$ & $\begin{array}{c}\mathrm{C} \\
119.45 \pm 1.37 \\
\end{array}$ & $\begin{array}{c}\mathrm{C} \\
115.31 \pm 2.44 \\
\end{array}$ & $\begin{array}{c}\mathrm{C} \\
109.28 \pm 2.21 \\
\end{array}$ & $\begin{array}{c}\mathrm{A} \\
150.08 \pm 1.53 \\
\end{array}$ & $\begin{array}{c}\text { A } \\
163.33 \pm 2.31 \\
\end{array}$ & $\begin{array}{c}\mathrm{A} \\
158.33 \pm 2.25 \\
\end{array}$ \\
\hline $\begin{array}{c}\text { Zinc } \\
(\mu \mathrm{g} / \mathrm{dl})\end{array}$ & $\begin{array}{c}\text { B } \\
85.21 \pm 1.32 \\
\end{array}$ & $\begin{array}{c}\text { B } \\
92.21 \pm 1.72 \\
\end{array}$ & $\begin{array}{c}\text { B } \\
90.33 \pm 1.89 \\
\end{array}$ & $\begin{array}{c}\mathrm{C} \\
88.61 \pm 1.41 \\
\end{array}$ & $\begin{array}{c}\mathrm{C} \\
87.55 \pm 1.31 \\
\end{array}$ & $\begin{array}{c}\mathrm{C} \\
83.21 \pm 2.33 \\
\end{array}$ & $\begin{array}{c}\mathrm{A} \\
102.24 \pm 1.33 \\
\end{array}$ & $\begin{array}{c}\mathrm{A} \\
107.23 \pm 2.12 \\
\end{array}$ & $\begin{array}{c}\mathrm{A} \\
105 \pm 1.65 \\
\end{array}$ \\
\hline $\begin{array}{c}\text { Iron } \\
(\mu \mathrm{g} / \mathrm{dl})\end{array}$ & $\begin{array}{c}\text { B } \\
142.03 \pm 1.36\end{array}$ & $\begin{array}{c}\text { B } \\
138.21 \pm 1.57\end{array}$ & $\begin{array}{c}\text { B } \\
135.21 \pm 2.15\end{array}$ & $\begin{array}{c}\mathrm{C} \\
118.13 \pm 1.35\end{array}$ & $\begin{array}{c}\mathrm{C} \\
115.22 \pm 1.31\end{array}$ & $\begin{array}{c}\mathrm{C} \\
109 \pm 1.45 \\
\end{array}$ & $\begin{array}{c}\mathrm{A} \\
156.03 \pm 1.43\end{array}$ & $\begin{array}{c}\mathrm{A} \\
162.31 \pm 2.14\end{array}$ & $\begin{array}{c}\mathrm{A} \\
160.21 \pm 1.53\end{array}$ \\
\hline
\end{tabular}

For each week: Means within the same row of different litters are significantly different at $(\mathrm{P}<0.05)$. 
Table 3: Showing the effects of administration of therapeutic dose $(0.4 \mathrm{mg} / \mathrm{kg} . \mathrm{bw}$.) and over dose (1 $\mathrm{mg} / \mathrm{kg} . \mathrm{bw})$ ) of non steroidal anti-inflammatory Piroxicam ${ }^{\circledR}$ in serum of breeding rabbit on macro and micro minerals (Mean \pm S.E.).

\begin{tabular}{|c|c|c|c|c|c|c|c|c|c|}
\hline \multirow{3}{*}{ Parameters } & \multicolumn{9}{|c|}{ Dose and periods } \\
\hline & \multicolumn{3}{|c|}{ Therapeutic dose } & \multicolumn{3}{|c|}{ Over dose } & \multicolumn{3}{|c|}{ Control } \\
\hline & $2^{\text {nd }}$ week & $4^{\text {th }}$ week & $8^{\text {th }}$ week & $2^{\text {nd }}$ week & $4^{\text {th }}$ week & 1 & $2^{\text {nd }}$ week & $4^{\text {th }}$ week & ek \\
\hline $\begin{array}{c}\begin{array}{c}\text { Total protein } \\
(\mathrm{gm} / \mathrm{dL})\end{array} \\
\end{array}$ & $\begin{array}{c}\text { B } \\
7.45 \pm 0.31\end{array}$ & $\begin{array}{c}\text { B } \\
7.32 \pm 0.22 \\
\end{array}$ & $\begin{array}{c}\text { B } \\
7.13 \pm 0.21 \\
\end{array}$ & $\begin{array}{c}\text { A } \\
8.14 \pm 0.1 \\
\end{array}$ & $\begin{array}{c}\mathrm{A} \\
7.95 \pm 0.09 \\
\end{array}$ & $\begin{array}{c}\mathrm{A} \\
8.35 \pm 0.21 \\
\end{array}$ & $\begin{array}{c}\mathrm{A} \\
7.59 \pm 0.20 \\
\end{array}$ & $\begin{array}{c}\text { A } \\
8.31 \pm 0.33\end{array}$ & $\begin{array}{c}\text { A } \\
7.92 \pm 0.42\end{array}$ \\
\hline $\begin{array}{l}\text { Glucose } \\
(\mathrm{mg} / \mathrm{dL})\end{array}$ & $\begin{array}{c}\text { A } \\
55.34 \pm 1.98 \\
\end{array}$ & $\begin{array}{c}\mathrm{A} \\
56.35 \pm 2.33\end{array}$ & $\begin{array}{c}\mathrm{A} \\
55.31 \pm 1.88 \\
\end{array}$ & $\begin{array}{c}\mathrm{A} \\
55.28 \pm 2.52\end{array}$ & $\begin{array}{c}\mathrm{A} \\
58.17 \pm 1.78\end{array}$ & $\begin{array}{c}\mathrm{A} \\
56.31 \pm 2.30\end{array}$ & $\begin{array}{c}\mathrm{A} \\
55.45 \pm 0.76 \\
\end{array}$ & $\begin{array}{c}\text { A } \\
55.31 \pm 0.67\end{array}$ & $\begin{array}{c}\text { A } \\
56.21 \pm 0.88\end{array}$ \\
\hline $\operatorname{ALP}(g / d L)$ & $\begin{array}{c}\text { B } \\
95.24 \pm 1.31 \\
\end{array}$ & $\begin{array}{c}\text { B } \\
98.21 \pm 1.37 \\
\end{array}$ & $\begin{array}{c}\text { B } \\
97.34 \pm 1.91 \\
\end{array}$ & $\begin{array}{c}\mathrm{A} \\
131.5 \pm 1.26 \\
\end{array}$ & $\begin{array}{c}\mathrm{A} \\
141.31 \pm 2.08 \\
\end{array}$ & $\begin{array}{c}\mathrm{A} \\
138.34 \pm 1.57 \\
\end{array}$ & $\begin{array}{c}\text { B } \\
97.72 \pm 0.4 \\
\end{array}$ & $\begin{array}{c}\text { B } \\
98.58 \pm 0.61 \\
\end{array}$ & $\begin{array}{c}\text { B } \\
102.23 \pm 0.9 \\
\end{array}$ \\
\hline AST (I.U./L) & $\begin{array}{c}\text { B } \\
98.31 \pm 3.81 \\
\end{array}$ & $\begin{array}{c}\text { B } \\
95.34 \pm 3.71 \\
\end{array}$ & $\begin{array}{c}\mathrm{B} \\
92.21 \pm 4.11 \\
\end{array}$ & $\begin{array}{c}\mathrm{A} \\
265.09 \pm 3.62 \\
\end{array}$ & $\begin{array}{c}\mathrm{A} \\
259.31 \pm 4.31 \\
\end{array}$ & $\begin{array}{c}\mathrm{A} \\
255.31 \pm 4.21 \\
\end{array}$ & $\begin{array}{c}\mathrm{B} \\
94.05 \pm 5.11 \\
\end{array}$ & $\begin{array}{c}\mathrm{B} \\
101.32 \pm 4.68\end{array}$ & $\begin{array}{c}\mathrm{B} \\
98.17 \pm 4.23\end{array}$ \\
\hline ALT (I.U./L) & $\begin{array}{c}\text { B } \\
30.14 \pm 2.88 \\
\end{array}$ & $\begin{array}{c}\text { B } \\
32.44 \pm 3.11 \\
\end{array}$ & $\begin{array}{c}\text { B } \\
38.21 \pm 1.77 \\
\end{array}$ & $\begin{array}{c}\mathrm{A} \\
59.98 \pm 3.92 \\
\end{array}$ & $\begin{array}{c}\mathrm{A} \\
60.66 \pm 2.47 \\
\end{array}$ & $\begin{array}{c}\mathrm{A} \\
62.41 \pm 2.98 \\
\end{array}$ & $\begin{array}{c}C \\
26.49 \pm 3.13 \\
\end{array}$ & $\begin{array}{c}\mathrm{C} \\
28.56 \pm 2.28 \\
\end{array}$ & $\begin{array}{c}\mathrm{C} \\
29.34 \pm 3.15 \\
\end{array}$ \\
\hline Urea $(\mathrm{gm} / \mathrm{dL})$ & $\begin{array}{c}\text { B } \\
21.31 \pm 1.34 \\
\end{array}$ & $\begin{array}{c}\mathrm{A} \\
27.31 \pm 1.55 \\
\end{array}$ & $\begin{array}{c}\mathrm{A} \\
29.25 \pm 1.81 \\
\end{array}$ & $\begin{array}{c}\mathrm{A} \\
30.06 \pm 1.23 \\
\end{array}$ & $\begin{array}{c}\mathrm{A} \\
32.13 \pm 2.12 \\
\end{array}$ & $\begin{array}{c}\mathrm{A} \\
33.14 \pm 1.89 \\
\end{array}$ & $\begin{array}{c}\text { B } \\
22.38 \pm 0.85 \\
\end{array}$ & $\begin{array}{c}\mathrm{B} \\
24.25 \pm 0.93 \\
\end{array}$ & $\begin{array}{c}\text { B } \\
26.25 \pm 0.81 \\
\end{array}$ \\
\hline $\begin{array}{r}\text { Creatinine } \\
(\mathrm{mg} / \mathrm{dL}) \\
\end{array}$ & $\begin{array}{c}\text { B } \\
1.12 \pm 0.12\end{array}$ & $\begin{array}{c}\text { B } \\
1.29 \pm 0.09\end{array}$ & $\begin{array}{c}\text { B } \\
1.93 \pm 0.21 \\
\end{array}$ & $\begin{array}{c}\mathrm{A} \\
3.18 \pm 0.1 \\
\end{array}$ & $\begin{array}{c}\text { A } \\
3.42 \pm 0.52 \\
\end{array}$ & $\begin{array}{c}\mathrm{A} \\
3.94 \pm 0.33 \\
\end{array}$ & $\begin{array}{c}\text { B } \\
1.09 \pm 0.09\end{array}$ & $\begin{array}{c}\text { B } \\
1.11 \pm 0.09\end{array}$ & $\begin{array}{c}\text { B } \\
1.21 \pm 0.13 \\
\end{array}$ \\
\hline $\begin{array}{c}\text { Cholesterol } \\
(\mathrm{mg} \%)\end{array}$ & $\begin{array}{c}\text { B } \\
160.21 \pm 3.03 \\
\end{array}$ & $\begin{array}{c}\text { B } \\
159.5 \pm 2.66\end{array}$ & $\begin{array}{c}\mathrm{B} \\
158.5 \pm 2.33 \\
\end{array}$ & $\begin{array}{c}\mathrm{A} \\
174.25 \pm 3.33 \\
\end{array}$ & $\begin{array}{c}\mathrm{A} \\
174.5 \pm 3.58 \\
\end{array}$ & $\begin{array}{c}\text { A } \\
176 \pm 4.03 \\
\end{array}$ & $\begin{array}{c}\text { B } \\
160.9 \pm 3.03 \\
\end{array}$ & $\begin{array}{c}\text { B } \\
160.3 \pm 2.58 \\
\end{array}$ & $\begin{array}{c}\text { B } \\
160 \pm 3.03 \\
\end{array}$ \\
\hline
\end{tabular}

For each week: Means within the same row of different litters are significantly different at $(\mathrm{P}<0.05)$. 
Table 4: Showing the effects of administration of therapeutic dose $((0.4$ $\mathrm{mg} / \mathrm{kg} . \mathrm{bw}$.$) and over dose (1 \mathrm{mg} / \mathrm{kg} . \mathrm{bw}))$ of non steroidal antiinflammatory Piroxicam ${ }^{\circledR}$ in testes homogenates in breeding rabbit on Alkaline phosphatase and zinc (Mean \pm S.E).

\begin{tabular}{|c|c|c|c|c|}
\hline $\begin{array}{c}\text { Periods of } \\
\text { sampling }\end{array}$ & $\begin{array}{c}\text { Parameters } \\
\text { measured }\end{array}$ & $\begin{array}{c}\text { Therapeutic } \\
\text { dose }\end{array}$ & $\begin{array}{c}\text { Double or over } \\
\text { dose }\end{array}$ & Control \\
\hline \multirow{2}{*}{$2^{\text {nd }}$ week } & $\begin{array}{c}\text { ALP } \\
(\mathrm{g} / \mathrm{dL})\end{array}$ & $\begin{array}{c}\mathrm{A} \\
11.3 \pm 0.33\end{array}$ & $\begin{array}{c}\mathrm{A} \\
10.12 \pm 0.12 \\
\end{array}$ & $\begin{array}{c}\text { B } \\
9.88 \pm 0.09\end{array}$ \\
\hline & $\begin{array}{c}\text { Zinc } \\
\mu \mathrm{g} / \mathrm{ml} \text { or gram }\end{array}$ & $\begin{array}{c}\text { B } \\
39.37 \pm 3.33\end{array}$ & $\begin{array}{c}\text { B } \\
39.37 \pm 3.39 \\
\end{array}$ & $\begin{array}{c}\mathrm{A} \\
55.73 \pm 3.57\end{array}$ \\
\hline \multirow{2}{*}{$4^{\text {th }}$ week } & $\begin{array}{l}\text { ALP } \\
\mathrm{g} / \mathrm{dL}\end{array}$ & $\begin{array}{c}\text { B } \\
11.45 \pm 0.52\end{array}$ & $\begin{array}{c}\mathrm{A} \\
13.25 \pm 2.53\end{array}$ & $\begin{array}{c}C \\
10.50 \pm 0.09\end{array}$ \\
\hline & $\begin{array}{c}\text { Zinc } \\
\mu \mathrm{g} / \mathrm{ml} \text { or gram }\end{array}$ & $\begin{array}{c}\mathrm{B} \\
36.83 \pm 2.63 \\
\end{array}$ & $\begin{array}{c}\text { B } \\
34.9 \pm 3.44 \\
\end{array}$ & $\begin{array}{c}\mathrm{A} \\
61.60 \pm 3.66 \\
\end{array}$ \\
\hline \multirow{2}{*}{$8^{\text {th }}$ week } & $\begin{array}{l}\text { ALP } \\
\text { g/dL }\end{array}$ & $\begin{array}{c}\text { B } \\
12.25 \pm 0.58 \\
\end{array}$ & $\begin{array}{c}\mathrm{A} \\
14.75 \pm 0.76 \\
\end{array}$ & $\begin{array}{c}\mathrm{C} \\
8.50 \pm 0.06 \\
\end{array}$ \\
\hline & $\begin{array}{c}\text { Zinc } \\
\mu \mathrm{g} / \mathrm{ml} \text { or gram }\end{array}$ & $\begin{array}{c}\text { B } \\
40.37 \pm 3.7\end{array}$ & $\begin{array}{c}\mathrm{C} \\
35.57 \pm 2.57 \\
\end{array}$ & $\begin{array}{c}\text { A } \\
52.7 \pm 2.57\end{array}$ \\
\hline
\end{tabular}

For each week: Means within the same row of different litters are significantly different at $(\mathrm{P}<0.01)$.

Table 5: Showing the effects of administration of therapeutic dose $((0.4$ $\mathrm{mg} / \mathrm{kg}$.bw.) and over dose $(1 \mathrm{mg} / \mathrm{kg} . \mathrm{bw}))$ of Non steroidal antiinflammatory Piroxicam ${ }^{\circledR}$ in serum of breeding rabbit on testosterone and prostaglandin F2 $\alpha(\mathrm{PGF} 2 \alpha)($ Mean \pm S.E).

\begin{tabular}{|c|c|c|c|c|}
\hline $\begin{array}{l}\text { Periods of } \\
\text { sampling }\end{array}$ & $\begin{array}{c}\text { Parameters } \\
\text { Measured }\end{array}$ & $\begin{array}{c}\text { Therapeutic } \\
\text { dose }\end{array}$ & $\begin{array}{c}\text { Double or over } \\
\text { dose }\end{array}$ & Control \\
\hline \multirow{2}{*}{$2^{\text {nd }}$ week } & $\begin{array}{c}\text { Testosterone } \\
\mathrm{ng} / \mathrm{dL}\end{array}$ & $\begin{array}{c}\mathrm{C} \\
318.22 \pm 5.33 \\
\end{array}$ & $\begin{array}{c}\mathrm{A} \\
342.33 \pm 5.67 \\
\end{array}$ & $\begin{array}{c}\text { B } \\
332.66 \pm 5.67 \\
\end{array}$ \\
\hline & $\begin{array}{c}\text { PGF2 } \alpha \\
\mathrm{Pg} / \mathrm{ml}\end{array}$ & $\begin{array}{c}\text { B } \\
311.33 \pm 4.89 \\
\end{array}$ & $\begin{array}{c}\mathrm{C} \\
307.66 \pm 5.88 \\
\end{array}$ & $\begin{array}{c}\mathrm{A} \\
360.76 \pm 6.33\end{array}$ \\
\hline \multirow{2}{*}{$4^{\text {th }}$ week } & $\begin{array}{c}\text { Testosterone } \\
\mathrm{ng} / \mathrm{dL}\end{array}$ & $\begin{array}{c}\mathrm{C} \\
324.33 \pm 5.88 \\
\end{array}$ & $\begin{array}{c}\text { A } \\
387.67 \pm 6.33 \\
\end{array}$ & $\begin{array}{c}\mathrm{B} \\
334.53 \pm 5.33 \\
\end{array}$ \\
\hline & $\begin{array}{c}\text { PGF2 } \alpha \\
\mathrm{Pg} / \mathrm{ml}\end{array}$ & $\begin{array}{c}\text { B } \\
278.67 \pm 4.67 \\
\end{array}$ & $\begin{array}{c}\mathrm{A} \\
252.33 \pm 5.88 \\
\end{array}$ & $\begin{array}{c}\mathrm{A} \\
353.33 \pm 5.67 \\
\end{array}$ \\
\hline \multirow{2}{*}{$8^{\text {th }}$ week } & $\begin{array}{c}\text { Testosterone } \\
\text { ng/dL }\end{array}$ & $\begin{array}{c}\mathrm{C} \\
330.67 \pm 7.33 \\
\end{array}$ & $\begin{array}{c}\mathrm{A} \\
490.33 \pm 7.67 \\
\end{array}$ & $\begin{array}{c}\mathrm{B} \\
355.66 \pm 5.67\end{array}$ \\
\hline & $\begin{array}{c}\text { PGF2 } \alpha \\
\mathrm{Pg} / \mathrm{ml}\end{array}$ & $\begin{array}{c}\text { B } \\
208.33 \pm 5.67\end{array}$ & $\begin{array}{c}\mathrm{C} \\
156.33 \pm 4.67\end{array}$ & $\begin{array}{c}\mathrm{A} \\
346.67 \pm 5.88 \\
\end{array}$ \\
\hline
\end{tabular}

For each week: Means within the same row of different litters are significantly different at $(\mathrm{P}<0.01)$. 


\section{DISCUSSION}

Non-steroidal anti-inflammatory Drugs (NSAIDs); chemically heterogeneous large groups of drugs which suppress inflammation in a manner similar to steroids, but with less side effects of sedation, respiratory depression, or addiction than steroids. They are widely used for the treatment of inflammatory disorders and painful conditions such as rheumatoid arthritis, gout, bursitis, painful menstruation, and headache in human. They are effective in the relief of pain and fever. Ando and Lombardino (1983); Dorigo et al. (2010). NSAIDs inhibit the cyclooxygenase (COX) activity resulting in decreased synthesis of prostaglandin, leukotriene and thromboxane precursors such as the ubiquitous enzyme which catalyzes the initial step in the synthesis of prostanoids. Prostanoid is any of a group of C-20 fatty acids complex with an internal five or six carbon rings such as prostaglandins, prostanoic acid, prostacyclins, and thromboxane; derived from arachidonic acid (C-20 polyunsaturated fatty acid with four cis double bonds). The action or the synthesis of prostanoids are involved in the modulation of a variety of pathophysiologic processes including inflammation, hemostasis, thrombosis, cytoprotection, ulceration, hemodynamic and other the progression of kidney diseases Lanas et al. (2003). Thus, NSAIDs as non-selective inhibitors of the cyclooxygenases (both the cyclooxygenase-1 and cyclooxygenase-2 enzymes) may have beneficial as well as untoward effects on a variety of diseases Berenguer, et al. (2002); Gilhotra et al. (2009) Piroxicam is used in the treatment of osteoarthritis, dysmenorrhoea, pyrexia and as an analgesic Ando and Lomardino (1983). Piroxicam selectively inhibit cyclooxygenase-2 over cyclooxygenase-1 Grosman (2007). The results indicated that there is a significant decrease in RBCs, $\mathrm{Hb}$ and PCV values. These results become very obvious when the Piroxicam injected by over dose. Also the results showed significant decrease in total WBCs and neutrophils when injected the Piroxicam by over dose and at the therapeutic dose all the periods of experiment. These results agreed with those recorded by Otterness et al. (1982) who recorded that the piroxicam inhibit the edema and total leucocytes and mononuclear infiltration Abe et al. (1983) illustrated that piroxicam posses prominent efficiency on allergic inflammation and may function on several activities of inflammatory cells.

The decreasing level of serum calcium, phosphorus, magnesium, copper, zinc and iron values that observed by injection of over dose and both lower than that of control dose of Piroxicam. These results agreed 
with those of Basha et al. (2011) where they used six oxicams, sudoxicam, isoxicam, Piroxicam, tenoxicam, meloxicam and lornoxicam, and compared in an attempt to understand why, despite close chemical structures, two of them were associated with an increased risk of toxicity in patients. Different factors have been revealed which may explain these differences. A weak association constant to serum albumin (HSA), together with a high plasma concentration, favors a rapid increase in unbound concentration $(\mathrm{Cu})$ when total plasma concentration rises (peak of absorption). Pathological states may enhance this increase when both HAS plasma concentrations is decreased and free fatty acid concentrations are increased. However, the main cause of toxicity may be the existence in some subjects of HSA natural mutants whose ability to bind oxicams is markedly lower than normal But Vanderschueren et al. (1991) found that the treatment with low dose of piroxicam was of no influence on the observed changes in Ca., Ph., levels the significant increase in the activities of AST, ALT and ALP and Kidney function tests that showed a significant increase in both urea and creatinine specially in over dose treatment group. In groups treated with therapeutic dose no significant change in AST, ALP, Urea and creatinin levels that agreed with findings of Goker et al. (1999) Also, the cholesterol level decrease in treated group with therapeutic dose all over the period that agreed with Sedigheh et al. (2005) who concluded that piroxicam was found to reduce C- Reactive protein (CRP), triglesried and LDL-C. it also lead to an increase in ant-oxidant capacity and HDL-C.

Testosterone significantly decreased in group treated with therapeutic dose these agreed with Martini et al. (2008) conclude that piroxicam can exert detrimental effects up on reproductive physiology (Fertilization reproductive hormone levels and cyclooxigenas inhibition) which depends on the dose and /or the drug employed.

In conclusion study revealed that the treatment with piroxicam negative hematological changes, decrease in some serum elements $\mathrm{Ca}$., Ph., Mag., Cu., Zn., and Fe.) with subsequent substandard and function nephro- and hepato- toxicities specially with the overdose of drug

These results also indicated that, using of Piroxicam at its therapeutic dose $(0.4 \mathrm{mg} / \mathrm{kg}$.Bw $)$ gave its best anti-inflammatory results in rabbits and other animals. So it could be recommended that piroxicam should be applied only as necessary by dose level not exceed than $(0.4 \mathrm{mg} / \mathrm{kg}$. Bw) to avoid its haemato -nephro and hepato toxicities and to avoid element deficiency by overdose of drug treatment. 


\section{REFERENCES}

Abe, N.; Tanaka, K.; Kanaoka, K.; Eyawa, M.; Watanabe, I. and Hirais (1983): Action of piroxicam on allergic inflammation Nibbon yakurigaku Zasshi, may, 81(5): 431-40.

Adams, J.E.; Schechtman, K.B.; Landt, Y.; Ladenson, J.H. and Jaffe, A.S. (1994): Comparable detection of acute myocardial infarction by CK-MB iso-enzyme and cardiac troponin-I. Clin. Chem. 40: 1291-5.

Ando, GA. and Lombardino, JG. (1983): Piroxicam A literature review of new results from laboratory and clinical studies Eur. J. Rheumatol. Inflamm. 6(1): 12-23.

AOAC. (1975): Association of official analytical chemists. Official methods of analysis $12^{\text {th }}$ ed., Washington, DC.

Basha, R.; Baker, C.H.; Sankpal, U.T.; Ahmad, S.; Safe, S.; Abbruzzese, J.L. and Abdelrahim, M. (2011): Therapeutic applications of NSAIDS in cancer: special emphasis on tolfenamic acid. Front Biosci (Schol Ed). 1; 3: 797-805.

Berenguer, B.; Alarcon Delalastra, C.; Moveno, F.J. and Martin, MJ. (2002): Chronic gastric ulcer healing in rats subjected to selective and non selective Cyclootxygenase-2 inhibitors Eur. J. Pharmacology. 3, 442(1-2): 125-135.

Bradshaw, D.; Cashin, C.H.; Kennedy, A.J. and Roberts, N.A. (1984): Pharmacological and biochemical activities of tenoxicam (Ro 12-0068), a new non-steroidal anti-inflammatory drug. Agents Actions. 15 (5-6): 569-77.

Coulombe, J.J. and Favreau, L. (1963): A new simple semi micro method for calorimetric determination of urea. Clin.Chem.9: 102-108.

Dorigo, MT.; Doro, D. and Cecchinato, A. (2010): Aqueous prostaglandin E2 and intraocular pressure after argon laser trabeculoplasty In glaucoma patients pretreated with topical piroxicam. Brain Behavimmun, 24(3): 409-19.

Duncan, D.B. (1955): Multiple range and multiple F test biometrics (11): 1-42.

Gilhotra, RM.; Gilhotra, N. and Mishra, DN. (2009): Piroxicam bio adhesive ocular insert physico shemical characterization and evaluation in prostaglandin in duced inflammation Curr Eye Res. 34(12): 1.65-73. 
Goker, B.; Yrtsever, E. and Sener, A. (1999): Effect of tenoxicam on biochemical serum parameters of rats Drug Metabolism, Drug Interact., 15(2-3): 207-213.

Grosman, N. (2007): Comparison of the influence of NSAIDs with different COX-selectivity on histamine release from mast cells isolated from naïve and sensitized rats. Int Immunopharmacol. 7(4): 532-40.

Heeb, HL.; Chun, R.; Koch, DE.; Moore, L.; Radlinsky, M.; Corse, M.; Pellerin, MA.; Garrett, L. and Hunter, RP. (2005): Multiple dose pharmacokinetics and acute safety of piroxicam and cimetidine in the cat. J. Vet. Pharmacol Ther. 28(5): 447-52.

Kind, P.R.N. and King, E.G. (1954): Colorimetric determination of Alkaline phosphatase activity. J. Clinic. Path. 6: 322.

Kocaoğlu, S.; Karan, A.; Berkan, T.; Başdemir, G. and Akpinar, R. (1997): Urinary gamma-glutamyl transferase activity in rats with nonsteroidal anti-inflammatory drug-induced nephrotoxicity. Arch Immunol Ther Exp (Warsz). 45(1): 73-7.

Lanas, A.; Jimenez, P.; Ferrandez, A.; Escartin, A.; Arenas, J.; Estera, F. and Ortego, J. (2003): Selective cox-2 inhibition is associated with decreased mucosal damage induced by acid and pepsin in rabbit esophagitis. Inflammation, 27(1): 21-9.

Lobetti, RG. and Joubert, KE. (2000): Effect of administration of nonsteroidal anti-inflammatory drugs before surgery on renal function in clinically normal dogs. Am. J. Vet Res. 61(12): 1501-7.

Martini, AC.; Vincenti, LM.; Santillán, ME.; Stutz, G.; Kaplan, R. and Ruiz RD, de Cuneo MF. (2008): Chronic administration of nonsteroidal-antiinflammatory drugs (NSAIDS): effects upon mouse reproductive functions. Rev. Fac. Cien. Med. Univ. Nac. Cordoba. 65(2): 47-59.

Otterness, I.G.; Larson, D.L. and Lombardino, J.G. (1982): An analyses of piroxicam models of arthritis Agent actions Jul. 12 (3): 308-12.

Peters, T. (1968): Colorimetric method for determination of total serum proteins. Clin.Chem., 14: 1147-1153.

Reitman, S. and Frankel, S. (1957): A colorimetric method for determination of serum glutamic oxaloacetic and glutamic pyurvic transaminase. Am. J. Path., 26: 1-13.

Ritland, S.R. and Gendler, S.J. (1999): Chemoprevention of intestinal adenomas in the ApcMin mouse by piroxicam: kinetics, strain 
effects and resistance to chemo suppression. Carcinogenesis. 20(1): 51-8.

SAS. (2006): Statistical analysis system. SAS USER's Guid .

Schrenk, W.G. (1975): Analytical atomic spectroscopy plenum press. Neo York \& London.

Sedigheh, Asgary; Gholam-Ali. Nadri; Gholam-Rezadashti, Zamzam and Paknahad (2005): Effect of ant-inflammatory drug, Piroxicam, on some biochemical factors and development of experimental atherosclerosis in rabbits ARYA J. Spring Vol.1, Issue I

Sigurdardottir, S.L.; Freysdottir, J.; Vikingsdottir, T.; Valdimarsson, H. and Vikingsson, A. (2008): Do non-steroidal anti-inflammatory drugs influence chronic inflammation? The effects of Piroxicam on chronic antigen-induced arthritis in rats. Scand J. Rheumatol. 37(6): 469-76.

Trinder, P. (1969): Determination of glucose in blood using glucose oxidase with an alternative oxygen acceptor. Ann. Clin. L. I. N. Biochem. 6, 24.

Vanderschueren, D.; Gevers, G.; Dequeker, J.; Geusens, P.; Nijs, K.; Devos, P.; Deroo, M. and Bouillol, R. (1991): Seasonal variation in bone metabolism in young healthy subject Calcify. Tissue Int., 49(2): 84-9.

Vane, J.R. and Botting, R.M. (1997): Mechanism of action of aspirinlike drugs. Semin Arthritis, Rheum. 26(6 Suppl 1): 2-10.

Williams, R.B.; Carylel, W.; Bond, D. and Brown, I. (1999): The efficacy and economic benefits of paracox, alive attenuated anticoccidial vaccine in commercial trialy with 29341-29355. 
Assiut Vet. Med. J. Vol. 57 No. 131 October 2011 
Assiut Vet. Med. J. Vol. 57 No. 131 October 2011 
Assiut Vet. Med. J. Vol. 57 No. 131 October 2011 